\title{
ON THE DECOMPOSITION OF THE $s$-RADICAL OF A NEAR-RING
}

\author{
by J. F. T. HARTNEY
}

(Received 27th October 1987)

\begin{abstract}
This paper concerns a Jacobson-type radical for the near-ring $N$. This radical, denoted by $J_{s}(N)$ has an external representation on a type- $0 \mathrm{~N}$-group of a very special kind. Such $N$-groups are said to be of type-s. The main objective of this paper is to decompose $J_{s}(N)$ as a sum $J_{s}(N)=J_{1 / 2}(N)+A+B$ for $N$ satisfying the descending chain condition for $N$-subgroups. In this decomposition $J_{1 / 2}(N)$ is nilpotent and $A$ is the unique minimal ideal modulo which $J_{s}(N)$ is nilpotent.
\end{abstract}

1980 Mathematics subject classification (1985 Revision): 16-XX.

\section{Introduction}

Throughout this article our near-rings will be assumed to be zero symmetric and right distributive. If the near-ring $N$ satisfies the descending chain condition for left ideals $(N$ subgroups) we say that $N$ satifies the $D C C L$ (respectively, $D C C N$ ). Our direct sums will always be direct sums of $N$-kernels (kernels of $N$-homomorphisms). Hence, $\Omega=\bigoplus_{j \in J} \Omega_{j}$ for the $N$-group $\Omega$ will always mean that $\Omega_{j}$ is an $N$-kernel of $\Omega$ for each $j \in J$. The following theorem which is referred to as the Main Theorem is proved.

Let $N$ be a near-ring which satisfies the $D C C N$. If the s-radical $J_{s}(N)$ is not zero, then there exists an ideal $A$ of $N$ contained in $J_{s}(N)$ such that $A$ is uniquely minimal amongst all ideals $B$ of $N$ for which $J_{s}(N / B)$ is non-zero and nilpotent. Moreover $J_{s}(N / A)=J_{s}(N) / A$.

The main theorem is then used to decompose the s-radical as $J_{s}(N)=J_{1 / 2}(N)+A+B$, where $A$ is the unique minimal ideal of the main theorem and $B$ is an ideal which is nilpotent modulo an ideal $C$ to be defined in the sequel.

This generalises the work initiated in [5]. If the near-ring $N$ is itself nilpotent modulo some ideal $C$, then $J_{1 / 2}(N / C)=J_{0}(N / C)=J_{s}(N / C)$. To avoid this trivial situation we will assume throughout that the near-ring $N$ is not nilpotent modulo any ideal $C \neq N$. That is, we assume throughout that $N^{k} \geq C$ for every positive integer $k$ and every ideal $C \neq N$. Also, $J_{s}(N) \neq N$ throughout this paper. We use as our main tool the nil-rigid series of a near-ring $N$ first defined and discussed by S. D. Scott [9]. Scott's generalized version of his original work appears in [10]. The length of the nil-rigid series throws some light on the manner in which the $s$-radical decomposes with $J_{1 / 2}(N)$ as a summand (not 
necessarily a direct summand) but many problems remain. We refer the reader to Meldrum [6] and Pilz [8] for the basic definitions and results used in this paper.

\section{Preliminaries}

The following results are of great importance in the development of the theory in subsequent sections. Proofs of these reults can be found in $[4,6]$ and also in [1].

Theorem 2.1. Let $N$ be a near-ring which satisfies the DCCL. Then the factor $N$-group $N-J_{1 / 2}(N)=\bigoplus_{i=1}^{k} \Omega_{i}$, where $\Omega_{i}$ is an $N$-group of type-0 for $i=1, \ldots, k$.

Lemma 2.2. Let $\Omega=\bigoplus_{i \in I} \Omega_{i}$ be an $N$-group, where each $\Omega_{i}$ is an $N$-group of type-0. Then any $N$-kernel $\Delta$ of $\Omega$ is a direct summand of $\Omega$ with a direct sum of some of the $\Omega_{i}$ as a co-summand. That is $\Omega=\bigoplus_{j \in J \leqq I} \Omega_{j} \oplus \Delta$.

Details of the theory involving the $s$-radical, $J_{s}(N)$ of a near-ring $N$ can be found in [2] and [6]. We give a summary of some of the results which we will require later.

An $N$-group $\Omega$ of type- 0 is said to be of type-s if for all $\omega \in \Omega$ for which $N \omega \neq(0)$ we have

(i) $N \omega=\bigoplus_{i \in I} \Omega_{i}$, where each $\Omega_{i}$ is of type-0

and

(ii) there exists $\omega^{\prime} \in N \omega$ such that $N \omega=N \omega^{\prime}$ and the annihilating left ideal $(0: \omega)$ is equal to the annihilating left ideal $\left(0: \omega^{\prime}\right)$.

An ideal $A$ of $N$ is said to be $s$-primitive if it is the annihilator of some $N$-group of type-s. $J_{s}(N)$ is the intersection of all $s$-primitive ideals of $N$. If $N$ has no $s$-primitive ideals, we define $J_{s}(N)$ to be $N$ itself. A left ideal $L$ of $N$ is said to be $s$-modular if the factor $N$-group $N-L$ is of type-s.

We have the inclusions $J_{0}(N) \leqq J_{1 / 2}(N) \leqq J_{s}(N) \leqq J_{1}(N)$. The following theorem can be proved using Theorem 2.1 and Lemma 2.2.

Theorem 2.3. If $N$ is a near-ring satisfying the $D C C L$, then any ideal containing $J_{1 / 2}(N)$ is an intersection of s-modular left ideals.

From Theorem 2.3 it follows that $J_{s}(N)$ is the smallest two-sided ideal containing $J_{1 / 2}(N)$ in the $D C C L$ case. Indeed if $N$ satisfies the $D C C L$, then $J_{s}(N)$ may be defined as the smallest two-sided ideal which is an interesection of 0 -modular left ideals (cf. [2]).

We now give a brief description of an ideal of $N$ which is in some sense dual to $J_{s}(N)$. For further details we refer the reader to [3].

Let $\mathscr{F}$ be the collection of all ideals $A$ of $N$ which are of the form $A=\bigoplus_{i \in I} N e_{i}$, where

(i) $N e_{i}$ is of type-0 and $e_{i} \in N e_{i}$ for each $i \in I$ 
and

(ii) $e_{i}^{2}=e_{i}$ and $e_{i} e_{j}=0$ if $i>j$ for some ordering on the index set $I$.

An element of $\mathscr{F}$ is said to be $\mathscr{F}$-decomposable or to have an $\mathscr{F}$-decomposition.

Using Zorn's lemma one can show that if $\mathscr{F} \neq \emptyset$, then it possesses a unique maximal element which we call the socle-ideal of $N$ and which we denote by $\operatorname{Soi}(N)$. If $\mathscr{F}=\emptyset$ we define $\operatorname{Soi}(N)$ to be the zero ideal. If $N$ satisfies the $D C C L$, then $\operatorname{Soi}(N)$ has an $\mathscr{F}$ decomposition $\operatorname{Soi}(N)=\bigoplus_{i=1}^{k} N e_{i}$, with $\left\{e_{1}, \ldots, e_{k}\right\}$ an orthogonal indempotent set. Moreover we have

Theorem 2.4. [3] Let $N$ be a near-ring wich satisfies the $D C C L$. Then $N=\operatorname{Soi}(N) \oplus L$ where $L$ is a left ideal containing $J_{1 / 2}(N)$.

Theorem 2.5. [3] Let $N$ be a near-ring which satisfies the $D C C L$. Then $J_{s}(N)$ is the unique smallest ideal amongst all ideals $B$ of $N$ for which $\operatorname{Soi}(N / B)=N / B$. Moreover, $J_{s}(N)=(0)$ if and only if $\operatorname{Soi}(N)=N$.

\section{Proof of the main theorem}

We begin by giving an outline of the concept of a nil-rigid series for the near-ring $N$.

Let $\operatorname{nil}(N)$ denote the nil radical of the near-ring $N$. An ideal $A$ of $N$ is said to be rigid if whenever $B$ is an ideal of $N$ contained in $A$, then

$$
(A / B) \cap \operatorname{nil}(N / B)=(0) \text {. }
$$

In [9] Scott showed that there is a unique maximal rigid ideal in $N$ which he calls the crux of $N$ and which we will denote by $\operatorname{Crux}(N)$. In $[10], \operatorname{Crux}(N)$ is arrived at via a more general route. It emerges as a semi-simple part corresponding to the Baer lower radical.

Definition. For the near-ring $N$ satisfying the ascending chain condition for ideals, let $L_{1}=\operatorname{nil}(N)$ and let $C_{1}$ be the ideal containing $L_{1}$ such that

$$
C_{1} / L_{1}=\operatorname{Crux}\left(N / L_{1}\right)
$$

Further, let $L_{2}$ be the ideal containing $C_{1}$ such that

$$
L_{2} / C_{1}=\operatorname{nil}\left(N / C_{1}\right)
$$

If $\alpha$ is a non-limit ordinal define $L_{\alpha}$ to be the ideal of $N$ containing $C_{\alpha-1}$ such that

$$
L_{\alpha} / C_{\alpha-1}=\operatorname{nil}\left(N / C_{\alpha-1}\right)
$$

and $C_{\alpha}$ to be the ideal containing $L_{\alpha}$ such that

$$
C_{\alpha} / L_{a}=\operatorname{Crux}\left(N / L_{\alpha}\right)
$$


If $\alpha$ is a limit ordinal, define

$$
C_{\alpha}=\bigcup_{\beta<\alpha} C_{\beta}
$$

and

$$
L_{\alpha}=\bigcup_{\beta<\alpha} L_{\beta} .
$$

The transfinite ascending sequence of ideals.

$$
\{0\}, L_{1}, C_{1}, L_{2}, C_{2}, \ldots
$$

is called the nil-rigid series of the near-ring $N$.

For the near-ring $N$ we state the following facts concerning $\operatorname{Crux}(N)$ and nil-rigid series.

(a) $\operatorname{Soi}(N) \leqq \operatorname{Crux}(N)$ and if $N$ satisfies the $D C C N$, then $\operatorname{Crux}(N)-\operatorname{Soi}(N)$, [3].

(b) For near-rings $N$ satisfying the $D C C N^{*} \operatorname{Soi}(N)=\operatorname{Crux} N$ and $\operatorname{nil}(N)=J_{0}(N)$. In this case $\operatorname{Crux}(N)$ and $\operatorname{nil}(N)$ cannot be simultaneously zero. Thus in this case nilrigid series are strictly ascending $[10]$.

(c) If $N$ satisfies the $D C C N$, then the nil-rigid series of $N$ is finite and there exists a positive integer $\alpha$ such that $C_{\alpha}=N$. Recall that $N$ is not nilpotent modulo any proper ideal; hence the series cannot stop at an $L_{\beta}$ for any $\beta$.

The positive integer $\alpha$ is called the nil-rigid length or the nil-rigid class number of $N$ if $C_{\alpha}=N$.

Lemma 3.1. Let $N$ be a near-ring satisfying the DCCN and let $\alpha$ be its nil-rigid length. The s-radical $J_{s}(N)$ is nilpotent if and only if $\alpha=1$.

Proof. $J_{s}(N)$ nilpotent implies that $J_{s}(N)=J_{0}(N)$ and hence by Theorem 2.5 $\operatorname{Crux}\left(N / J_{0}\right)=\operatorname{Soi}\left(N / J_{s}\right)=N / J_{s}$. It follows that the nil-rigid series for $N$ is

$$
L_{1}=J_{0}(N)=J_{s}(N), \quad N=C_{1} .
$$

Conversely, $\alpha=1$ implies $N / J_{0}=C_{1} / J_{0}=\operatorname{Soi}\left(N / J_{0}\right)$ and again by Theorem 2.5 we have $J_{0}(N)=J_{s}(N)$. Hence $J_{s}(N)$ is nilpotent.

We note that if $N$ has nil-rigid length $\alpha=1$ and $J_{s}(N) \neq(0)$, then the main theorem is true for $N$. We need only prove the main theorem for $\alpha>1$. For such cases $J_{s}(N) \neq(0)$ by Lemma 3.1 .

Lemma 3.2. Let $N$ be a near-ring which satisfies the DCCL. For any ideal $A$ of $N$, $J_{s}(N / A)=\left(J_{s}(N)+A\right) / A$. Moreover, $J_{s}(N / A)$ is nilpotent if and only if $\left(J_{s}(N)\right)^{m} \leqq A$ for some positive integer $m$, depending on $A$. 
Proof. By Theorem $2.3 J_{s}(N)+A$ is an intersection of $s$-modular left ideals of $N$ and hence $\left(J_{s}(N)+A\right) / A$ is an intersection of $s$-modular left ideals of $N / A$. Thus $\left(J_{s}(N)+A\right) / A \geqq J_{s}(N / A)$. On the other hand, the intersection of all $s$-modular left ideals of $N$ containing $A$ also contains $J_{s}(N)+A$ and it follows that $J_{s}(N / A) \geqq\left(J_{s}(N)+A\right) / A$.

Hence we have equality

$$
J_{s}(N / A)=\left(J_{s}(N)+A\right) / A \text {. }
$$

Lemma 3.3. Let $N$ be a near-ring satisfying the DCCN. If in the nil-rigid series of $N$ we have $L_{\alpha}<C_{\alpha}=N$ and $\alpha>1$, then $J_{s}\left(N / C_{\alpha-1}\right)$ is non-zero and nilpotent. Moreover, $\left(J_{s}(N)\right)^{m} \leqq C_{\alpha-1}$ for some positive integer $m$.

Proof. $\quad C_{\alpha}=N$ implies that $\operatorname{Soi}\left(N / L_{\alpha}\right)=N / L_{\alpha}$. Hence by Theorem $2.5, L_{\alpha} \geqq J_{s}(N)$ and we have

$$
J_{0}\left(N / C_{\alpha-1}\right)=L_{\alpha} / C_{\alpha-1} \geqq\left(J_{s}(N)+C_{\alpha-1}\right) / C_{\alpha-1}=J_{s}\left(N / C_{\alpha-1}\right) .
$$

Thus $J_{s}\left(N / C_{\alpha-1}\right)=J_{0}\left(N / C_{\alpha-1}\right)$ is nilpotent.

Since the nil-rigid series of $N$ is strictly ascending $L_{\alpha} \neq C_{\alpha-1}$ and we have that $J_{s}\left(N / C_{a-1}\right) \neq(0)$.

Lemma 3.4. Let $N$ be a near-ring which satisfies the DCCN. Suppose that in the nil-rigid series for $N$ we have $L_{\alpha}<C_{\alpha}=N$ and $\alpha>1$. Then there exists an ideal $A$ of $N$ such that $A$ is uniquely minimal amongst all ideals $B$ of $N$ for which $J_{s}(N / B)$ is non-zero and nilpotent. Moreover,

$$
A \leqq J_{s}(N) \cap C_{\alpha-1} \text { and } J_{s}(N / A)=J_{s}(N) / A .
$$

Proof. Let $\xi=\left\{B: B\right.$ an ideal of $N, J_{s}(N / B)$ is nilpotent $\}$. By Theorem $3.3 \xi \neq \emptyset$. Also, if $B \in \xi$ put $C=J_{s}(N) \cap B$. Then since $\left(J_{s}(N)\right)^{m} \leqq B$ for some positive integer $m$ (Lemma $3.2)$, it follows that $\left(J_{s}(N)\right)^{r} \leqq C$ for some positive integer $r$ and hence $C \in \xi$. Since $N$ satisfies the $D C C N, \xi$ has a minimal element $A$, say. By the above observation we may assume that $A$ is contained in $J_{s}(N)$. If $D \in \xi$, then by the above $D \cap A \in \xi$ so that $A \leqq D$ by the minimality of $A$. Thus the ideal $A$ is uniquely minimal in $\xi$. Clearly, by Lemmas 3.2 and $3.3 J_{s}(N / A)=\left(J_{s}(N)\right) / A$ and we see that $A \leqq J_{s}(N) \cap C_{\alpha-1}$. Finally, we show that $J_{s}(N / A) \neq(0)$, that is $J_{s}(N) \neq A$. If $J_{s}(N)=A$, then from the proof of Lemma 3.3, we have

$$
L_{\alpha} / C_{\alpha-1}=\left(J_{s}(N)+C_{\alpha-1}\right) / C_{\alpha-1}=\left(A+C_{\alpha-1}\right) / C_{a-1}=C_{\alpha-1} / C_{\alpha-1}=0 .
$$

This contradicts the fact that the nil-rigid series for $N$ is strictly ascending. Thus $J_{s}(N) \neq A$.

Corollary. If $J_{s}(N) \neq(0)$, then it cannot be idempotent; that is $\left(J_{s}(N)\right)^{2} \neq J_{s}(N)$. 
We remark that Lemma 3.4 is false in the case of the radicals $J_{1}(N)$ and $J_{2}(N)$. For suppose $N$ is a near-ring which satisfies the $D C C N$ and let $J_{s}(N)=(0)$. Then $N=\operatorname{Soi}(N)$ is idempotent and hence if $J_{1}(N) \neq(0)$, it is idempotent.

The main theorem, stated in the introduction, can now immediately be deduced from Lemmas 3.1 and 3.4.

Definition. The unique minimal ideal $A$ of the main theorem will be called the s-socle.

We conclude this section by discussing some of the properties of the s-socle.

Theorem 3.5. Let $N$ be a near-ring with $D C C N$ and suppose that in the nil-rigid series for $N$ we have $L_{\alpha}<C_{\alpha}=N$ with $\alpha>1$. Then the s-socle is not contained in $L_{\alpha-1}$.

Proof. Let $A$ be the $s$-socle of $N$. If $\alpha=2$, then $L_{\alpha-1}=L_{1}=J_{0}(N)$ and $A \leqq L_{1}=J_{0}(N)$ implies that $J_{s}(N)$ is nilpotent.

But by Lemma $3.1 J_{s}(N)$ is nilpotent if and only if $\alpha=1$. Thus $A$ is not contained in $L_{1}$ and the theorem is true for $\alpha=2$. Now suppose that $\alpha>2$. If $A \leqq L_{\alpha-1}$, then since $\left(J_{s}(N)\right)^{m} \leqq A$ for some positive integer $m$ and $\left.L_{\alpha-1} / C_{\alpha-2}\right)$ is a nilpotent ideal of $N / C_{\alpha-2}$ we have that $\left(J_{s}(N)\right)^{r} \leqq C_{\alpha-2}$ for some positive integer $r$. Thus

$$
J_{0}\left(N / C_{\alpha-2}\right)=L_{\alpha-1} / C_{\alpha-2} \geqq\left(J_{s}(N)+C_{\alpha-2}\right) / C_{\alpha-2}=J_{s}\left(N / C_{\alpha-2}\right) \text {. }
$$

It follows that $C_{\alpha-1} / L_{\alpha-1}=\operatorname{Soi}\left(N / L_{\alpha-1}\right)$ so that $N=C_{\alpha-1}$, contradicting the strict ascendency of the nil-rigid series of $N$. This proves the theorem.

Now $J_{0}(N) \cap \operatorname{Soi}(N)=(0)$ for otherwise $J_{0}(N)$ would contain an idempotent $e \neq 0$, appearing in the $\mathscr{F}$-decomposition of Soi $(N)$. In fact, in [3] it is shown that $J_{1 / 2}(N) \cap \operatorname{Soi}(N)=(0)$. Clearly, Soi $(N) \oplus J_{0}(N)$ has an $\mathscr{F}$-decomposition modulo $J_{0}(N)$. Thus $\operatorname{Soi}(N) \oplus J_{0}(N) \leqq C_{1}$, where $C_{1}$ is the ideal in the nil-rigid series of $N$ such that $\operatorname{Soi}\left(N / J_{0}(N)\right)=C_{1} / J_{0}(N)$. Consequently we have the following theorem which follows immediately from Theorem 3.5.

Theorem 3.6. Let $N$ be a near-ring with $D C C N$ such that in the nil-rigid series for $N, L_{\alpha}<C_{\alpha}=N$ with $\alpha>2$. Then the s-socle is not contained in $\operatorname{Soi}(N)$.

Thus Theorem 3.6 tells us that, in order to find examples of near-rings for which the $s$-socle is contained in $\operatorname{Soi}(N)$ we need look no further than near-rings with nil-rigid class number equal to 2 . Indeed, from Theorem 3.5 and the fact that the $s$-socle is contained in $C_{\alpha-1}$ we can immediately deduce the following theorem.

Theorem 3.7. Let $N$ be a near-ring which satisfies the DCCN and suppose that in the nil-rigid series for $N$ we have $L_{\alpha}<C_{\alpha}=N$. Then the s-socle $A$ of $N$ is contained in $C_{1}$ if and only if $\alpha=2$. 
The following theorem provides us with a class of near-rings in which the $s$-socle is always contained in $\operatorname{Soi}(N)$.

Theorem 3.8. Let $N$ be a near-ring with $D C C N$ and let the nil-rigid length of $N$ be 2 . If $\operatorname{Soi}(N) \oplus J_{0}(N)=C_{1}$, then the s-socle, $A$ of $N$ is contained in $\operatorname{Soi}(N)$.

Proof. Since $C_{a-1}=C_{1}$ we have $A \leqq C_{1}=\operatorname{Soi}(N) \oplus J_{0}(N)$. But $J_{0}(N)$ is a nilpotent ideal of $N$; hence $\operatorname{Soi}(N) \oplus J_{0}(N)$ is nilpotent modulo $\operatorname{Soi}(N)$. It follows that $A+\operatorname{Soi}(N)$ is nilpotent modulo $\operatorname{Soi}(N)$ and that $A^{h} \leqq \operatorname{Soi}(N)$ for some positive integer $h$. Thus $\left(J_{s}(N)\right)^{k} \leqq \operatorname{Soi}(N)$. But $A$ is uniquely minimal amongst all ideals $B$ of $N$ with the property that $\left(J_{s}(N)\right)^{r} \leqq B$ for some positive integer $r$. It follows that $A \leqq \operatorname{Soi}(N)$.

We remark that the equality $C_{1}=\operatorname{Soi}(N) \oplus \dot{J}_{0}(N)$ does not always hold in the case of near-rings $N$ with nil-rigid class number equal to 2 . We give the following example by way of illustration in which we use the representation theory developed in [5]. We note that, in the case of d.g. near-rings, with $D C C N$ the critical ideal, $\operatorname{Crit}(N)$ of [5] is precisely the socle ideal, Soi $(N)$.

Example. Let $V$ be a reduced free group on $m$ generators whose laws are precisely the universal laws of $S_{5}$, the symmetric group on 5 symbols. Take $m$ at least as great as the minimum number of generators for $S_{5}$. Let $N$ be the Neumann d.g. near-ring [7] associated with $V ; N$ is finite with an identity. Now $V-K \cong S_{5}$ for some normal subgroup $K$ of $V$ and so there is a left ideal $L$ such that $N-L$ is a faithful $N$-group. Moreover, every $N$-subgroup of $N-L$ is monogenic and there is a one-one lattice correspondence between the subgroups of $S_{5}$ and the $N$-subgroups of $N-L$. Under this correspondence the $N$-kernels of $N-L$ correspond to the normal subgroups of $S_{5}$. Now $S_{5}$ does not have a simple subgroup as a direct summand so that $N-L$ cannot have an $N$-group of type- 0 as a direct summand. By the theory in [5] $\operatorname{Crit}(N)=\operatorname{Soi}(N)$ annihilates $N-L$ and $\operatorname{Soi}(N)=(0)$ because $N-L$ is faithful. We note that the only simple groups which are homomorphic images of subgroups of $S_{5}$ and $A_{5}$ are $A_{5}$ itself and groups of prime order. Let $\Omega$ be the subgroup of $N-L$ which corresponds to $A_{5}$. Then $\bar{N}=N / J_{0}(N)$ is 0 -primitive with $\Omega$ a faithful $\bar{N}$-group. Also, $\operatorname{Soi}(\bar{N})=C_{1} / J_{0}(N)$ is idempotent and consists of a direct sum of copies of $\Omega$. Hence it does not annihilate $\Omega$. Now the $\bar{N}$-subgroups of $\Omega$ corresponding to subgroups of $A_{5}$ isomorphic to $A_{4}$ do not have $\bar{N}$-subgroups of type- 0 as direct summands and hence they are annihilated by Soi $(\bar{N})$. Let $\Delta$ be a faithful $\bar{N}$-group, where $\bar{N}=N / C_{1}$. Further, let $\Gamma$ be any type-0 $\bar{N}$-group which is a homomorphic image of an $\bar{N}$-subgroup of $\Delta$. Then by the above $\Gamma$ is of type-2 and we have $J_{s}(\bar{N})=J_{2}(\bar{N})=J_{0}(\bar{N})$.

Thus the nil-rigid series for $N$ is

$$
L_{1}=J_{0}(N), C_{1}, L_{2}, C_{2}=N
$$

where $L_{2} / C_{1}=J_{0}(\bar{N})$. We see that $\operatorname{Soi}(N) \oplus J_{0}(N)=J_{0}(N) \neq C_{1}$.

The following theorem is immediate. 
Theorem 3.9. If $N$ is a near-ring which satisfies the DCCN, then the s-socle $A$ is nilpotent, if and only if $J_{s}(N)$ is nilpotent.

Corollary 1. The s-socle $A$ is contained in $J_{0}(N)$ if and only if the nil-rigid class number of $N$ is 1 .

Corollary 2. If the s-socle of a near-ring is not zero, then it cannot be nilpotent.

Theorem 3.10. Let $N$ be a near-ring which satisfies the $D C C N$. Then $J_{1 / 2}(N)$ is contained in the s-socle $A$ if and only if $J_{1 / 2}(N)=(0)$.

Proof. If $J_{1 / 2}(N)=(0)$, then certainly $J_{1 / 2}(N) \leqq A$. In fact, $J_{1 / 2}(N)=J_{s}(N)$ by Theorem 2.3 so that $A=J_{1 / 2}(N)=(0)$. Conversely, if $J_{1 / 2}(N) \leqq A$, then by the remark following Theorem $2.3, J_{s}(N) \leqq A$. Hence $J_{s}(N)=A$ which is not possible if $J_{s}(N) \neq(0)$ by the main theorem. This proves the result.

Theorem 3.11. Let $N$ be a near-ring which satisfies the DCCN. Then the s-socle $A$ is contained in $J_{1 / 2}(N)$ if and only if the nil-rigid class number $\alpha$ of $N$ is 1 .

Proof. If $A \leqq J_{1 / 2}(N)$, then $J_{s}(N)$ is nilpotent so that $\alpha=1$ by Lemma 3.1 , conversely, $\alpha=1$ implies $J_{s}(N)$ is nilpotent so that $A=(0) \leqq J_{1 / 2}(N)$.

\section{A decomposition for $J_{s}(N)$}

We begin by characterizing the left ideals of the near-ring $N$ which contain $J_{1 / 2}(N)$. This will enable us to express $J_{1 / 2}(N / B)$, in terms of $J_{1 / 2}(N)$, where $B$ is any ideal of $N$.

Lemma 4.1. Let $N$ be a near-ring which satisfies the DCCL. Any left ideal $L$ of $N$ which contains $J_{1 / 2}(N)$ is an intersection of 0 -modular left ideals.

Proof. Since $L-J_{1 / 2}(N)$ is an $N$-kernel of $N-J_{1 / 2}(N)$ from Theorem 2.1 and Lemma 2.2 we have $N-L=\bigoplus_{i=1}^{k} \Delta_{i}$, where $\Delta_{i}$ is an $N$-group of type-0 for $i=1, \ldots, k$. Since $L$ contains $J_{1 / 2}(N)$ it is a modular left ideal $[2,8]$. That is, there exists $e \in N$ such that $n e-n \in L$ for all $n \in N$. Now the coset $e+L$ has a unique expression of the form

$$
e+L=\bar{e}_{1}+\bar{e}_{2}+\cdots+\bar{e}_{k}, \bar{e}_{i} \in \Delta_{i} \quad i=1, \ldots, k
$$

Putting $\bar{e}_{i}=e_{i}+L, i=1, \ldots, k$ we have for any $n \in N$

$$
\begin{aligned}
n+L=n e+L & =n\left(\bar{e}_{1}+\bar{e}_{2}+\cdots+\bar{e}_{k}\right) \\
& =n \bar{e}_{1}+n \bar{e}_{2}+\cdots+n \bar{e}_{k},
\end{aligned}
$$

because of the validity of left distribution over a direct sum of $N$-kernels. Thus we have 


$$
n+L=\left(n e_{1}+L\right)+\left(n e_{2}+L\right)+\cdots+\left(n e_{k}+L\right)
$$

It is clear that $\Delta_{i}$ is monogenic by $e_{i}+L$ for $i=1, \ldots, k$ and hence the annihilating left ideal $\left(0: \bar{e}_{i}\right)$ is 0 -modular. It follows readily that $L=\bigcap_{i=1}^{k}\left(0: \bar{e}_{i}\right)$.

Theorem 4.2. Let $N$ be a near-ring satisfying the DCCL and $B$ any ideal of $N$. Then $J_{1 / 2}(N / B)=\left(J_{1 / 2}(N)+B\right) / B$.

Proof. Clearly, $J_{1 / 2}(N / B) \geqq\left(J_{1 / 2}(N)+B\right) / B$. On the other hand, $J_{1 / 2}(N)+B$ is a left ideal containing $J_{1 / 2}(N)$ and hence it is an intersection of 0-modular left ideals of $N$, by Lemma 4.1.

Consequently, $\left(J_{1 / 2}(N)+B\right) / B$ is an intersection of 0 -modular left-ideals of the factor near-ring $N / B$ and hence must contain $J_{1 / 2}(N / B)$. The equality follows.

For the remainder of this section we will assume that the near-ring $N$ satisfies the $D C C N$ and has nil-rigid length $\alpha>1$. We note that $\operatorname{Soi}\left(N / L_{\alpha-1}\right)=\operatorname{Crux}\left(N / L_{\alpha-1}\right)=$ $C_{\alpha-1} / L_{\alpha-1}$. Also, $A$ will denote the $s$-socle throughout.

Now Theorem 2.4 tells us that

$$
N / L_{\alpha-1}=C_{\alpha-1} / L_{\alpha-1} \oplus L / L_{\alpha-1} \text {, where } \quad L_{\alpha-1} / C_{\alpha-2}=J_{0}\left(N / C_{\alpha-2}\right)
$$

if $\alpha>2, L_{1}=J_{0}(N)$ and $L / L_{\alpha-1}$ is a left ideal of $N / L_{\alpha-1}$ containing $J_{1 / 2}\left(N / L_{\alpha-1}\right)$. By Theorem 4.2 we have $J_{1 / 2}\left(N / L_{\alpha-1}\right)=\left(J_{1 / 2}(N)+L_{\alpha-1}\right) / L_{\alpha-1}$. From Lemma 2.2 we deduce that the factor near-ring $N / L_{\alpha-1}$ has the decomposition

$$
N / L_{\alpha-1}=\left(A+L_{\alpha-1}\right) / L_{\alpha-1} \oplus L^{\prime} / L_{\alpha-1} \oplus L / L_{\alpha-1},
$$

because $\left(A+L_{\alpha-1}\right) / L_{\alpha-1}$ is an $N$-kernel of $C_{\alpha-1} / L_{\alpha-1}$. Using the modular law we obtain the decomposition

$$
\begin{aligned}
J_{s}\left(N / L_{\alpha-1}\right) & =\left(J_{s}(N)+L_{\alpha-1}\right) / L_{\alpha-1} \\
& =\left[\left(A+L_{\alpha-1}\right) / L_{a-1}\right] \oplus\left[\left(J_{s}(N)+L_{a-1}\right) / L_{\alpha-1} \cap\left(L^{\prime} / L_{\alpha-1} \oplus L / L_{\alpha-1}\right)\right]
\end{aligned}
$$

Since the sum on the right is direct and $\left(J_{s}(N)\right)^{r} \leqq A$ for some positive integer $r$ it follows that

$$
\left(J_{s}(N)+L_{\alpha-1}\right) / L_{a-1} \cap\left(L^{\prime} / L_{\alpha-1} \oplus L / L_{\alpha-1}\right)
$$

is a nilpotent left ideal of $N / L_{\alpha-1}$ and hence is contained in $J_{1 / 2}\left(N / L_{\alpha-1}\right)$. But $J_{1 / 2}\left(N / L_{\alpha-1}\right) \leqq L / L_{\alpha-1}$ therefore the intersection (1) above is precisely $J_{1 / 2}\left(N / L_{\alpha-1}\right)$. We have shown that the following decomposition of $J_{s}\left(N / L_{a-1}\right)$ occurs.

$$
J_{s}\left(N / L_{\alpha-1}\right)=\left(A+L_{\alpha-1}\right) / L_{a-1} \oplus J_{1 / 2}\left(N / L_{\alpha-1}\right) \text {. }
$$


Thus we have proved the following theorem.

Theorem 4.3. Let $N$ be a near-ring which satisfies the DCCN and such that $N$ has nil-rigid class number $\alpha>1$. Then the s-radical $J_{s}(N)$ decomposes as

$$
J_{s}(N)=J_{1 / 2}(N)+A+B
$$

where $A$ is the s-socle of $N$ and $B=J_{s}(N) \cap L_{\alpha-1}$.

Corollary. If the near-ring $N$ has nil-rigid length $\alpha=2$, then

$$
J_{s}(N)=J_{1 / 2}(N)+A
$$

Proof. This follows immediately from

$$
L_{\alpha-1}=L_{1}=J_{0}(N) \leqq J_{1 / 2}(N) \text {. }
$$

Whether $B=J_{s}(N) \cap L_{\alpha-1} \leqq A+J_{1 / 2}(N)$ always implies that $\alpha=2$ is an open question. For $\alpha>1$ the equality $B=A+J_{1 / 2}(N)$ cannot hold for otherwise we would have a contradiction to Theorem 3.5 .

Since $\operatorname{Soi}(N)$ is contained in $C_{1}$ for any near-ring $N$, from Theorem 3.7 we must have $\alpha \leqq 2$ in order that the $s$-socle be contained in Soi $(N)$. By Theorem 2.7 of [3] an ideal is contained in $\operatorname{Soi}(N)$ if and only if its intersection with $J_{1 / 2}(N)$ is the zero ideal. From this it is easy to see that if $A \leqq \operatorname{Soi}(N)$, then $J_{s}(N)=A \oplus J_{1 / 2}(N)$. Thus we see that the decomposition given in Section 4 of [3] occurs in a relatively small class of near-rings.

We have seen that if $N$ is a near-ring with $D C C N$ and with nil-rigid class number $\alpha>1$, then $A \leqq J_{s}(N) \cap C_{a-1}$. We now investigate this relationship further.

Suppose $C$ is an ideal of $N$ with an $\mathscr{F}$-decomposition $C=\bigoplus_{i=1}^{s} N e_{i}$. Let $B \neq 0$ be an ideal of $N$ contained in $C$. Then any $b \in B$ has a unique expression of the form $b=n_{1} e_{1}+\cdots+n_{s} e_{s}$. Since the $e_{i}, i=1, \ldots, s$ form an orthogonal idempotent set we have that $b e_{i}=n_{i} e_{i}$ and hence $b=b e_{1}+\cdots+b e_{s}$. But $B$ is an ideal so tht each component $b e_{i}$ is in $B$ and $B=B e_{1} \oplus \cdots \oplus B e_{s}$. Now each $B e_{i}$ is an $N$-kernel of $N e_{i}$ and hence re-indexing if necessary, we have

$$
B=N e_{1} \oplus \cdots \oplus N e_{r}, \quad \text { for } \quad r \leqq s
$$

because each $N e_{i}$ is of type- 0 .

Thus we see that $B$ has an $\mathscr{F}$-decomposition with components from the summands in $C$.

Now let $N$ be a near-ring which satisfies the $D C C N$ and having nil-rigid class number $\alpha>1$. We have that $C_{\alpha-1}$ has an $\mathscr{F}$-decomposition modulo $L_{\alpha-1}$, that is $C_{\alpha-1} / L_{\alpha-1}=$ $\bigoplus_{i=1}^{m} \Delta_{i}$, where $\Delta_{i}$ is an $\left(N / L_{\alpha-1}\right)$-group of type-0 and $\Delta_{i}=N / L_{\alpha-1}\left(\bar{e}_{i}\right)$ with $\left\{\bar{e}_{1}, \ldots, \bar{e}_{m}\right\}$ an orthogonal idempotent set, $\bar{e}_{i}=e_{i}+L_{\alpha-1}, i=1, \ldots, m$.

By the previous discussion $\left(\left(J_{s}(N) \cap C_{\alpha-1}+L_{\alpha+1}\right) / L_{\alpha-1}\right.$ is $\mathscr{F}$-decomposable. Also 
$e_{i}+L_{\alpha-1} \in\left[\left(J_{s}(N) \cap C_{\alpha-1}\right)+L_{\alpha-1}\right] / L_{\alpha-1}$ implies that $e_{i}+L_{\alpha-1}=x+L_{\alpha-1}$, where $x \in J_{s}(N) \cap C_{\alpha-1}$.

We have that $e_{i}+L_{\alpha-1}=e_{i}^{m}+L_{\alpha-1}=x^{m}+L_{\alpha-1}$ for any positive integer $m$ and since $J_{s}(N)^{k} \leqq A$, where $A$ is the $s$-socle and $k$ some positive integer, it follows that $e_{i}+L_{\alpha-1} \in\left(A+L_{\alpha-1}\right) / L_{\alpha-1}$. Thus

$$
\left(\left(J_{s}(N) \cap C_{\alpha-1}\right)+L_{\alpha-1}\right) / L_{\alpha-1}=\left(A+L_{\alpha-1}\right) / L_{\alpha-1} .
$$

Thus we have proved the following theorem.

Theorem 4.4. Let $N$ be a near-ring satisfying the $D C C N$ and having nil-rigid class number equal to $\alpha>1$. Then $J_{s}(N) \cap C_{\alpha-1}=A+\left(J_{s}(N) \cap L_{\alpha-1}\right)$, where $A$ is the s-socle of $N$.

Corollary 1. If $\alpha=2$, then $J_{s}(N) \cap C_{\alpha-1}=J_{s}(N) \cap C_{1}=A+J_{0}(N)$.

Proof. $\quad L_{\alpha-1}=J_{0}(N)$ and $J_{s}(N) \cap J_{0}(N)=J_{0}(N)$.

Corollary 2. If $\alpha=2$ and $A \leqq \operatorname{Soi}(N)$, then $J_{s}(N) \cap \operatorname{Soi}(N)=A$.

Acknowledgements. This research was completed whilst I was a visitor at the Universities of Edinburgh and Nottingham. I thank the Anglo-American Corporation, the British Council and the Edinburgh Mathematical Society for every assistance given. I also wish to express my appreciation to Drs R. R. Laxton and J. D. P. Meldrum for their valuable comments.

\section{REFERENCES}

1. J. F. T. Hartney, Radicals and antiradicals in near-rings (Ph.D. thesis, University of Nottingham, 1979).

2. J. F. T. Hartney, A radical for near-rings, Proc. Roy. Soc. Edinburgh 93A (1982), 105-110.

3. J. F. T. Hartney, An antiradical for near-rings, Proc. Roy. Soc. Edinburgh 96A (1984), 185-191.

4. R. R. Laxton, Prime ideals and the ideal radical of a distributively generated near-ring, Math. Z. 83 (1964), 8-17.

5. R. L. Laxton and A. W. Machin, On the decomposition of near-rings, Abh. Math. Sem. Univ. Hamburg 38 (1972), 221-230.

6. J. D. P. Meldrum, Near-rings and their Links with Groups (Research Notes Math. 134, Pitman, 1985).

7. Hanna Neumann, On varieties of groups and their associated near-rings, Math. Z. 65 (1956), $36-69$.

8. Gunter Pilz, Near-rings (North-Holland Math. Studies 23, 1977). 
9. S. D. Scotr, Near-rings and Near-ring Modules (Ph.D. thesis, Australian National University, 1970).

10. S. D. Scott, Formation radicals for near-rings, Proc. London Math. Soc.. (3) 25 (1972), 441-464.

Department of Mathematics

UNIVERSITY OF THE WITWATERSRAND

Johannesburg, South Africa

2050 